\title{
RESULTADOS CLÍNICOS DE LA TERAPIA ESTOMATOLÓGICA EN PACIENTES CON MUCOSITIS ORAL POSTERIOR A QUIMIOTERAPIA Y RADIOTERAPIA: REPORTE DE DOS CASOS
}

\author{
${ }^{1}$ Martha Leonor Rebolledo Cobos, ${ }^{2}$ Jonathan Harris Ricardo, ${ }^{3}$ Antonio José Díaz Caballero. \\ ${ }^{1}$ Odontóloga Fundación Universitaria San Martín, Especialista en Estomatología y Cirugía Oral U. de Cartagena, Docente Fundación Universitaria San Martín, Cartagena (Colombia). \\ ${ }^{2}$ Odontólogo U. del Sinú, Especialista en Estomatología y Cirugía Oral U. de Cartagena, Docente Corporación Universitaria Rafael Núñez, Cartagena (Colombia). \\ ${ }^{3}$ Odontólogo U. de Cartagena, Especialista en Periodoncia U. Javeriana, Magíster en Educación U. del Norte, Doctorando en Ciencias Biomédicas U. de Cartagena, \\ Profesor titular U. de Cartagena, Cartagena (Colombia).
}

Autor responsable de correspondencia: Jonathan Harris Ricardo

Correo electrónico:j.harris.r@hotmail.com

\section{RESUMEN}

La mucositis es la inflamación y ulceración de todas las mucosas como consecuencia de tratamientos antineoplásicos como quimioterapia y radioterapia. En la cavidad bucal se manifiesta con dolor y ulceraciones que impiden la ejecución de las funciones orales básicas como la fonación, masticación y deglución siendo un factor de favorecimiento a la infección. Actualmente existen tratamientos multifactoriales van desde el alivio de síntomas y control de infecciones, hasta el restablecimiento de la mucosa oral, con resultados favorecedores en la calidad de vida del paciente oncológico. A continuación se presentan dos casos de pacientes que acudieron al departamento de estomatología del instituto oncológico Betania, en la ciudad de Barranquilla - Colombia con diagnóstico de mucositis bucal asociado a tratamiento antineoplásico, se estableció una terapéutica que mostró buenos resultados. [Rebolledo M, Ricardo JH, Díaz AJ. Resultados clínicos de la terapia estomatológica en pacientes con mucositis oral posterior a quimioterapia y radioterapia: reporte de dos casos. Ustasalud 2011; 10: 65 - 68 ]

Palabras clave: Estomatitis, Terapia combinada, Quimioterapia, Radioterapia.

\section{CLINICAL RESULTS OF STOMATOLOGICAL THERAPY IN PATIENTS WITH ORAL MUCOSITIS AFTER CHEMOTHERAPY AND RADIOTHERAPY: REPORT OF TWO CASES}

\begin{abstract}
Mucositis is the inflammation and ulceration of the mucous membranes due to antineoplastic treatments such as chemotherapy and radiotherapy. In the mouth, occurs with pain and ulcerations that impede the implementation of basic oral functions such as phonation, chewing and swallowing to be a factor favoring the infection. Currently there are multifactorial treatments, from relief of symptoms and infection control to the restoration of the oral mucosa. There are reports of drug therapies such as, antiseptic and anesthetic solutions, with results that favor the quality of life of cancer patients. Here are reported two cases of patients attending the department of dentistry of Bethany cancer institute in the city of Barranquilla - Colombia diagnosed with oral mucositis associated with antineoplastic therapy, for which treatment was established which provided good results.
\end{abstract}

Key words: Oral mucositis, Medication therapy management, Antineoplastic combined chemotherapy Protocols, Radiotherapy.

Recibido para publicación: 14 de abril de 2011. Aceptado para publicación: 15 de junio de 2011.

\section{INTRODUCCIÓN}

La mucositis se define como un proceso inflamatorio de las mucosas, como consecuencia de un tratamiento oncológico de quimioterapia y radioterapia. ${ }^{1}$ En la cavidad bucal se manifiesta generalizadamente a partir de la segunda semana del tratamiento antineoplásico, relacionado comúnmente con neoplasias malignas. $^{2}$

La fisiopatología de la mucositis bucal (MB) no está definida pero, es atribuible a la afección directa que producen los medicamentos oncológicos y el efecto ionizante de la radioterapia que interfiriere en el tiempo de recambio de las células epiteliales e induce a la apoptosis. ${ }^{3}$ Sonis y colaboradores establecieron un modelo fisiopatológico y afirmaron que la mucositis cursa por cuatro fases: una inflamatoria/vascular, fase epitelial, fase ulcerativa/bacteriana y una última fase de cicatrización. ${ }^{4}$ La primera manifestación clínica de la (MB) es el eritema con sensación de quemazón acompañado de cuadros febriles. De acuerdo con la severidad de los signos y síntomas, se clasifica en: Grado 0, donde no se evidencian signos, el paciente manifiesta sensación de quemazón en una zona localizada de la (MB), Grado I, leve eritema con sensibilidad, Grado II, moderada 
con ulceraciones aisladas menores de $1 \mathrm{~cm}$, Grado III, severa con ulceraciones generalizadas y Grado IV, edema severo, infecciones sobreagregadas, incapacidad para hablar, masticar y deglutir. ${ }^{5}$

El tratamiento de la (MB) es diverso ya que incluye la eliminación de factores irritantes y agravantes como placa bacteriana, cálculo dental, restauraciones defectuosas, bordes traumáticos, aplicación de crioterapia para inducir vasoconstricción, el uso de agentes protectores de mucosa, antibacterianos, antivirales, antifúngicos, antisépticos, para el control de infecciones, antiinflamatorios de tipo corticoesteroides, anestésicos locales para el alivio de la sintomatología dolorosa y sustancias reepitelizantes. ${ }^{6}$

De igual forma se utiliza el sucralfato debido a que genera una barrera citoprotectora al entrar en contacto con la mucosa ulcerada. ${ }^{7}$ El ácido retinoico (vitamina A) ejerce efectos significativos sobre la inflamación y la vitamina $\mathrm{E}$ que interfiere con el daño inflamatorio ocasionado por los radicales libres creados por la quimiorradioterapia; debido a estos planteamientos y a la diversidad de enfoque disponibles, se plantean las posibilidades de uso de estas terapéuticas para la prevención y tratamiento de la mucositis bucal, que mejoren la calidad de vida de los pacientes bajo tratamientos antineoplásicos. $^{8}$

\section{CASO 1}

Paciente masculino de 43 años de edad con diagnóstico de carcinoma mucoepidermoide en amígdala derecha, el cual acude al servicio de estomatología por presentar múltiples lesiones ulcerativas en la cavidad bucal debido al tratamiento antineoplásico instaurado (quimioterapia y radioterapia). El paciente manifiesta trismo, dolor y ardor generalizado en las mucosas orales, dificultad para masticar y deglutir alimentos, gingivorragia y disfonía.

En el examen clínico se evidencian múltiples ulceraciones de tamaño variable coalescentes, distribuidas en cara dorsal de lengua, mucosa yugal derecha e izquierda, paladar duro y blando, los cuales se observan sangrantes, con pseudoplacas de color amarillentas y dolor de moderado a severo, 15 días de evolución que determina un diagnóstico clínico de mucositis grado III (Figura 1).

Previo a la firma de un consentimiento informado se procedió a instaurar la terapéutica que se inicia con la administración de yodopolivinilpirrolidona $20 \mathrm{ml}$ cada 8 horas, como enjuague antiséptico, manteniéndolo en boca durante 30 segundos pre- vio a la higiene bucal, cambios en la dieta: consumir mayormente alimentos blandos y líquidos fríos, eliminar la ingesta de sustancias ácidas, saladas, bebidas y alimentos calientes y muy condimentados; posteriormente se indicó la aplicación de hielo local para inducir vasoconstricción de las lesiones, seguido a la aplicación en las zonas ulceradas una mezcla en partes iguales de lidocaína viscosa, hidróxido de aluminio en suspensión, betametasona clorhidrato $1 \mathrm{mg} / \mathrm{ml}$, nistatina en crema 1.000 .000 UI, aciclovir en crema 3 gr, 250.000 UI de vitamina A no ácida, vitamina E en grageas y una tableta triturada de sucralfato, aplicar esta mezcla en las zonas afectadas, con un hisopo estéril 2 veces al en 24 horas por 10 días. Para el alivio de la sintomatología dolorosa general se ordenó Ibuprofeno de 600 mg cada 4 horas por vía oral.

Al control de las lesiones que ocurrió a los 10 días de instaurada esta terapia, se observó desaparición de las lesiones ulcerativas en un $90 \%$, con ausencia de exudados inflamatorios y desaparición de sintomatología, igualmente se evidenció mejoría en la fonación, apertura bucal, masticación y deglución (Figura 2). Se indicó la continuidad de esta terapéutica por 5 días más para la consecuente desaparición de las lesiones.

\section{CASO 2}

Paciente masculino de 13 años de edad con diagnóstico de Leucemia Linfocitica Aguda, el cual acude al servicio de estomatología por presentar lesiones ulcerativas en mucosa labial inferior como consecuencia del tratamiento antineoplásico instaurado (quimioterapia intratecal). El paciente reporta dolor y ardor, dificultad para masticar, deglutir alimentos, hablar y realizarse higiene oral de forma habitual.

En el examen clínico se evidenciaron dos ulceraciones ubicadas en mucosa labial inferior, los cuales se eran sangrantes, circunscritas y con pseudoplacas amarillentas, xerostomía leve y dolor moderado con 14 días de evolución, con diagnóstico clínico de mucositis grado II (Figura 3). Previo a la firma de un consentimiento informado se procedió a instaurar la terapia que se menciona. Se inicia la terapéutica igual que el anterior caso con excepción de la inclusión de corticoesteroides en la mezcla de aplicación tópica debido a recomendación por parte del oncólogo, el cual se sustituyó con Diclofenaco al 3\% en gel.

A los 10 días de instaurada esta terapia, se observó resolución completa de las lesiones, con ausencia de exudados inflamatorios, reducción de la sintomatología, aumento en la capacidad para masticar, deglutir y hablar (Figura 4). 


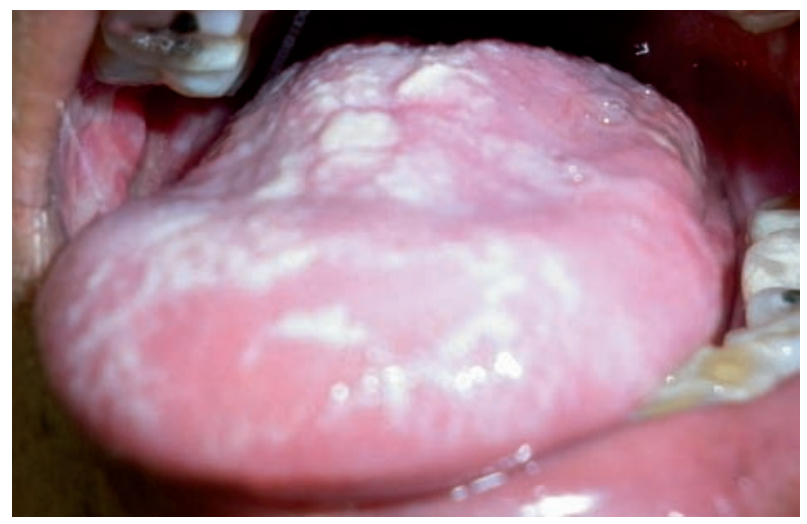

Figura 1. Se observan ulceraciones de tamaño variable en cara dorsal de lengua y mucosas yugales, con placa color blanquecina que denota una mucositis grado III.

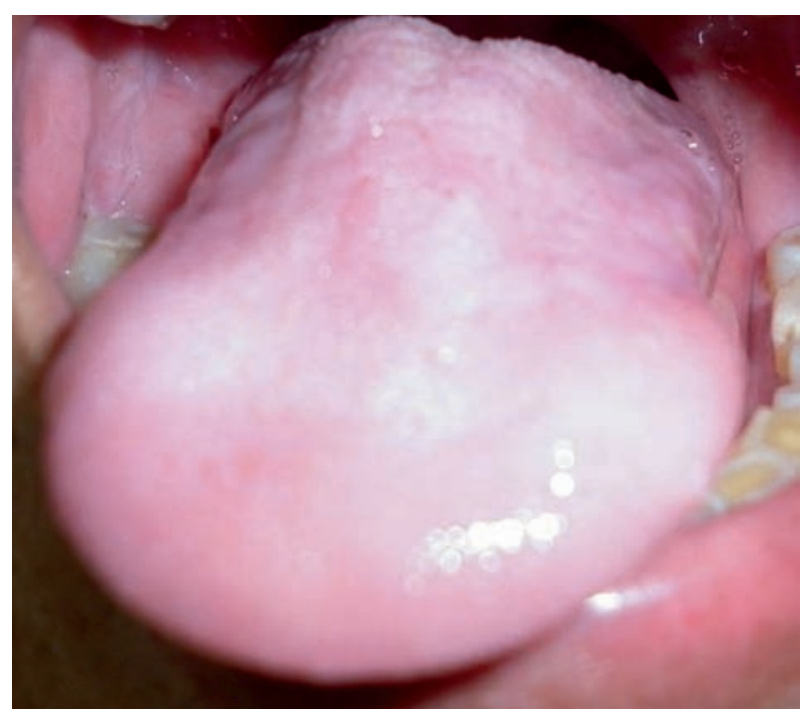

Figura 2. Control post-tratamiento a los 10 días donde se observa desaparición en un $90 \%$ de las lesiones ulcerativas por mucositis.

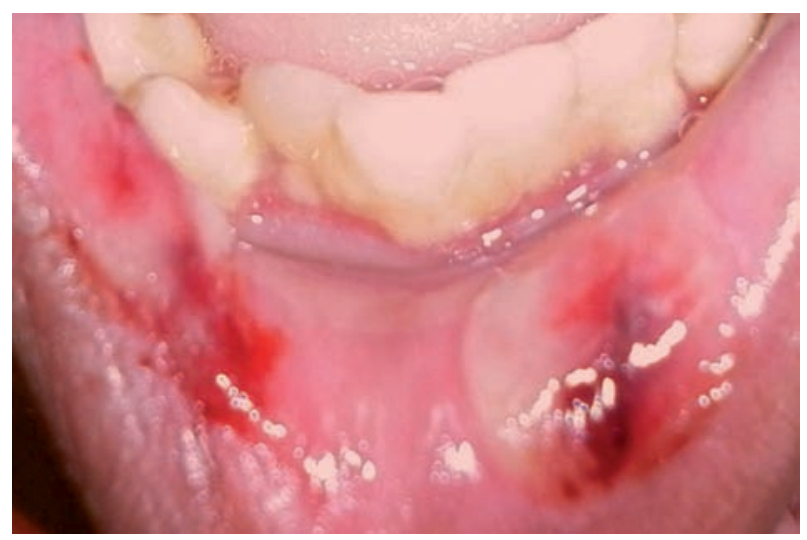

Figura 3. Se evidencian dos ulceraciones ubicadas en mucosa labial inferior, los cuales se muestran sangrantes, circunscritas y separadas, compatible con mucositis grado II.

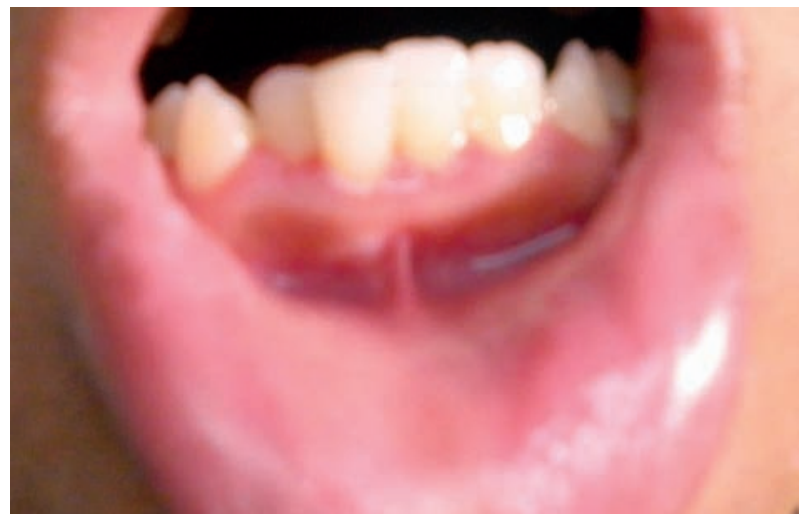

Figura 4. Control a los 10 días, se observa mejoría en un $100 \%$ de las lesiones por mucositis, mucosa totalmente sana.

\section{DISCUSIÓN}

El origen de la (MB) es atribuible a la acción citotóxica directa que ejercen los agentes quimio- radioterapéuticos sobre las células tanto tumorales y del tejido normal, que son de recambio rápido como lo es la mucosa bucal, que ocasiona estas lesiones ulcerativas. ${ }^{3}$ Sonis y colaboradores reportaron cuadro clínico de cuatro fases que cursa la (MB) iniciada desde una inflamatoria hasta una cicatrizal, aportan que la aparición de estas lesiones inicia a la segunda semana de tratamiento antineoplásico, semejante a los casos presentados en este reporte, donde las manifestaciones clínicas iniciales de (MB) se presentaron a la segunda semana de tratamiento y cursaron por las cuatro fases mencionadas. ${ }^{4}$

Dentro los diagnósticos más relacionados con (MB) se reportan leucemias, linfomas y tumores de cabeza y cuello, acertando con lo presentado en este reporte, donde un paciente presentó diagnóstico de leucemia linfocítica aguda y el segundo carcinoma mucoepidermoide de amígdala; se ha mencionado a la mucositis como la complicación bucal más común en pacientes que reciben quimioterapia. ${ }^{9,10}$

Rubenstein y colaboradores han sugerido que para el tratamiento de la (MB) un manejo integral a base de protectores de mucosa como el sucralfato e hidróxido de aluminio, antiinflamatorios tipo corticoesterorides, uso de agentes antimicóticos, antivirales, antibacterianos, analgésicos y anestésicos, incluida una terapia regenerativa con el uso de ácido retinoico y vitamina E. ${ }^{6}$ Tales planteamientos ofrecen buenas alternativas de curación de estas lesiones y mejoramiento de la calidad de vida del paciente iniciando desde la eliminación de factores irritantes como restauraciones desadaptadas, bordes cortantes y traumáticos e igualmente remoción de placa bacteriana y cálculo dental. ${ }^{10}$ Estos protocolos de manejo se tomaron en cuenta para el tratamiento de la (MB) en los pacientes, 
el cual constó de una mezcla por partes iguales de lidocaína viscosa, hidróxido de aluminio, betametasona clorhidrato, nistatina en crema, vitamina A, vitamina E y sucralfato, acompañado de la administración por vía oral de analgésicos, neutralización microbiana de la cavidad bucal con antiséptico, aplicación de hielo local para inducir vasoconstricción y recomendaciones acerca de higiene bucal como un adecuado cepillado dental, con resultados clínicos favorables a los 10 días de tratamiento, pues mejora de forma gradual la sintomatología, facilita la alimentación, deglución, fonación y aumenta significativamente su estado de salud frente a la enfermedad oncológica.

\section{BIBLIOGRAFÍA}

1. Feller L, Essop R, Wood NH, Khammissa RA, Chikte UM, Meyerov R, Lemmer J. Chemotherapy- and radiotherapyinduced oral mucositis: pathobiology, epidemiology and management. SADJ 2010; 65: 372 - 374.

2. Scully C, Epstein J, Sonis S. Oral mucositis: a challenging complication of radiotherapy, chemotherapy, and radiochemotherapy: part 1, pathogenesis and prophylaxis of mucositis. Head Neck 2003; 25: 1057 - 1070.

3. Sonis ST, Peterson DE, McGuire DB, Williams DA. Prevention of mucositis in cancer patients. J Natl Cancer Inst Monogr 2001; 29: 1-2.

4. Sonis ST. New thoughts on the initiation of mucositis. Oral Dis 2010; 16: 597 - 600.

5. Worthington HV, Clarkson JE, Bryan G, Furness S, Glenny $\mathrm{AM}$, Littlewood A, et al. Interventions for preventing oral mucositis for patients with cancer receiving treatment. Cochrane Database Syst Rev 2010; (12): CD000978.

6. Rubenstein EB, Peterson DE, Schubert M, Keefe D, McGuire $\mathrm{D}$, Epstein J. Clinical practice guidelines for the prevention and treatment or cancer therapy-induced oral and gastrointestinal mucositis. Cancer 2004; 100: 2026 - 2046.

7. Burkhart CG. Add sucralfate to list of aphthous ulcer treatments. Cutis 2002; 70: 275.

8. Lalla RV, Sonis ST, Peterson DE. Management of oral mucositis in patients who have cancer. Dent Clin North Am 2008; 52: 61 - 77.

9. Lerman MA, Laudenbach J, Marty FM, Baden LR, Treister NS. Management of oral infections in cancer patients. Dent Clin North Am 2008; 52: 129 - 153.

10. Keefe DM, Schubert MM, Elting LS, et al. Updated clinical practice guidelines for the prevention and treatment of mucositis. Cancer 2007; 109: 820 - 831.

Correos electrónicos de los autores:

Martha Leonor Rebolledo Cobos: malereco18@hotmail.com, Jonathan Harris Ricardo: j.harris.r@hotmail.com, Antonio José Díaz Caballero: antoniodiazc@yahoo.com

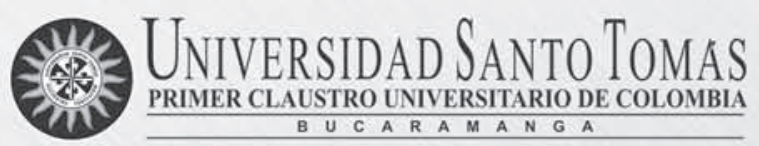

B U C A R A M A N G A

La Facultad de Odonto-

logía de la Universidad Santo Tomás en con-

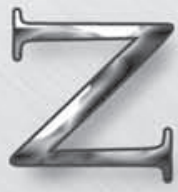

cordancia con la visión institucional aspira lograr:

Un compromiso efectivo de la comprensión renovada del hombre colombiano, su mundo

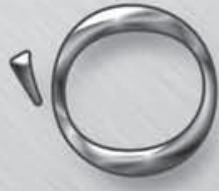

y sus valores, en un contexto de salud integral para todos; el reconocimiento nacional e internacional, como comunidad académica, a través de la investigación, la producción intelectual y el intercambio académico; liderazgo en los procesos relacionados con la salud bucal de la comunidad, a nivel regional y nacional; la cualificación permanente de sus servicios a la comunidad para responder efectiva y oportunamente a sus usuarios; el fortalecimiento permanente de su accionar pedagógico mediante el desarrollo humano integral, la aplicación de tecnologías de punta y la proyección comunitaria. 